\title{
COLOR HOLOGRAMS FOR WHITE LIGHT RECONSTRUCTION*
}

(diffuse reflectors; emulsion interference filter; E/T) J. Upatnieks, J. Marks, and R. Fedorowicz
Institute of Science and Technology The University of Michigan Ann Arbor, Michigan (Received 16 March 1966)
The similarity of Lippman color photography ${ }^{1}$ to the wavefront reconstruction method of Gabor was noted by Denisyuk, ${ }^{2}$ who showed that by using Lippman's method to record a hologram, the reconstruction could be made in white light. Subsequent related work has been reported by van Heerden, ${ }^{3}$ Pennington and Lin, ${ }^{4}$ Friesem, ${ }^{5}$ Fleisher et al. $^{6}$ and Hoffman, et al. ${ }^{7}$

We report the construction of holograms made from three-dimensional, diffusely reflecting objects, with reconstruction in full color in ordinary white light derived from a point source, such as, for example, the zirconium arc or an ordinary flashlight.

The hologram-making arrangement is shown in Fig. 1, which is identical to that used by Hoffman et al. ${ }^{7}$ The arrangement is conventional except for the introduction of the reference beam from the back surface of the photographic plate. Under this circumstance, the interference between signal and

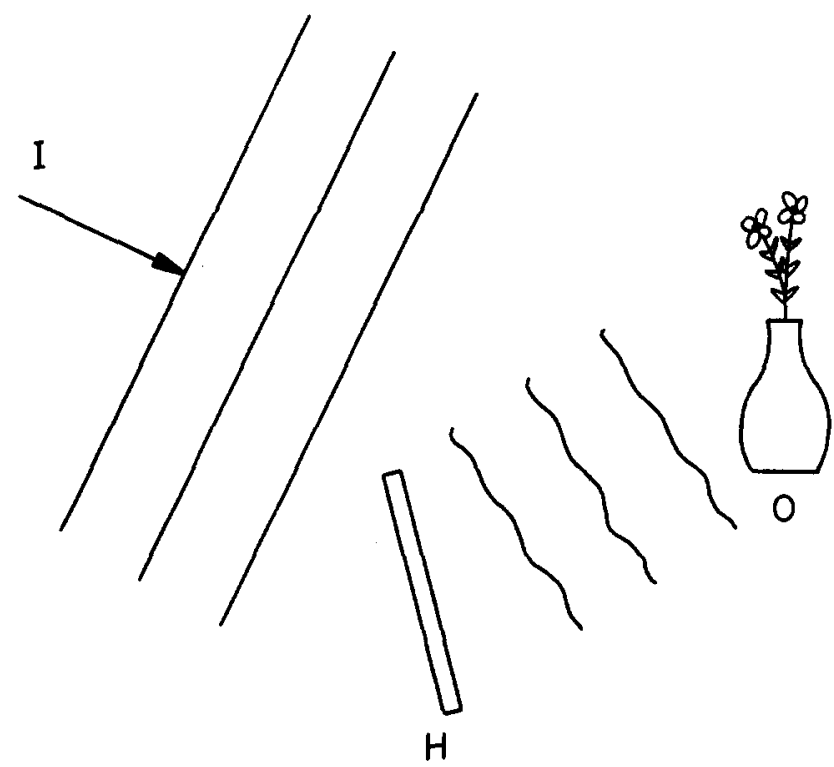

Fig. 1. Construction of the hologram. $O$, object; $H$, hologram; $I$, incident coherent light.

*This work was performed at the Willow Run Laboratories and sponsored by the Battelle Development Corporation, Columbus, Ohio. reference beam forms surfaces within the emulsion, spaced a distance $\lambda / 2^{\circ}$ apart, and lying roughly parallel to the emulsion surface, as shown in Fig. 2. The number of surfaces depends upon the thickness of the emulsion; for standard Eastman-Kodak 649 plates, the emulsion thickness is about $16 \mu$, thus giving rise to about 50 recorded surfaces.

In the reconstruction process (Fig. 2), the hologram may be illuminated with white light from a point source. Each surface reflects a portion of the incident light; light from the various surfaces produces reinforcement for that wavelength used in making the hologram. The hologram thus acts as its own interference filter, producing in reflected light the usual holographic image. For an emulsion

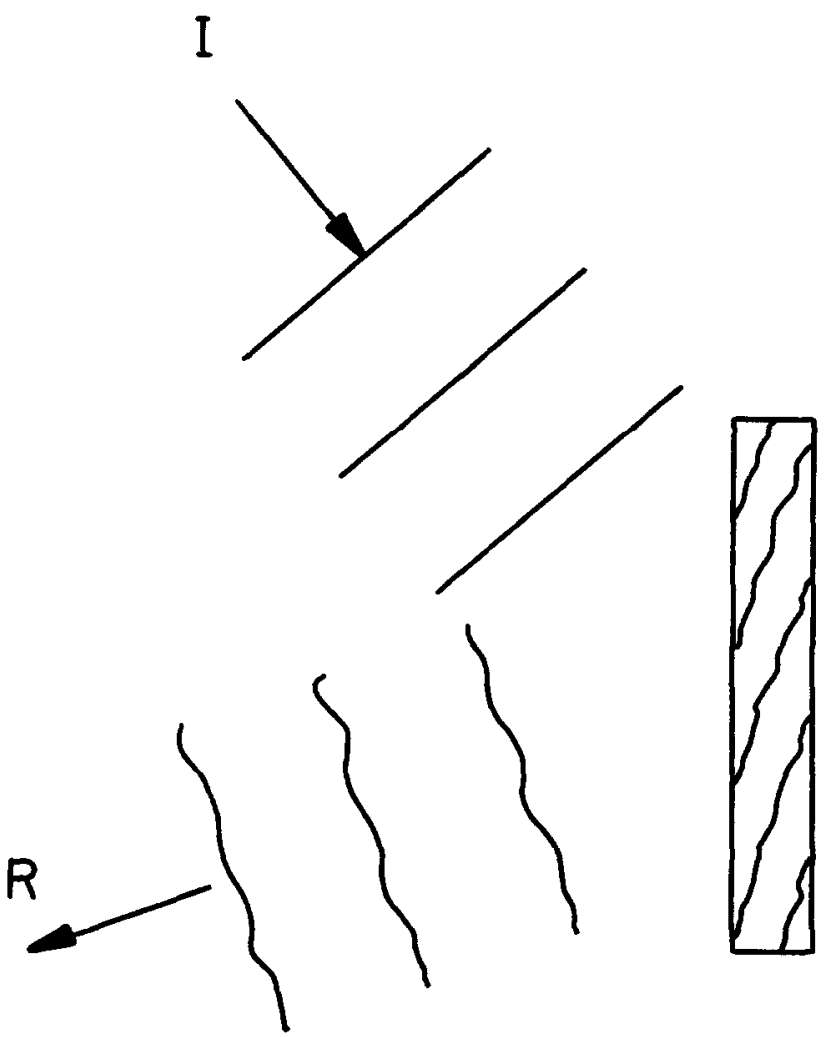

Fig. 2. The reconstruction process. The hologram is illuminated with white light. The fringe surfaces within the emulsion reflect the wavelength component in which the hologram was made. $I$ is incident light, $R$ is reflected light. 
thickness of $15 \mu$, the hologram, considered as an interference filter, has a reflection bandwidth of about $100 \AA$. Thus the reconstructed image lacks sharpness, although specially-coated thick emulsions can alleviate this shortcoming.

By making the hologram with light in three primary color wavelengths, (the $6328 \AA$ line of the helium-neon laser and the $5145 \AA$ and $4880 \AA$ lines of the argon ion laser) we have achieved a full color image. The image is illuminated with three primary colors and the reference beam similarly consists of three colors, an arrangement similar to that of Pennington and Lin, ${ }^{4}$ except for the reverse direction of the reference beam. Each of the three spectral components produces its own re corded interference pattern.

In the reconstruction process, each fringe pattern reflects light of the same wavelength that generated the fringe pattern. Thus, an image in full color is produced in light reflected from the hologram.

Shrinkage of the emulsion in the developing and fixing process has caused difficulty. This shrinkage, which amounts to about $13 \%$ of the initial thickness, causes a spectral shift in the reflection passband. Thus, a hologram made in red reconstructs in green, etc., and a reconstruction in full color is precluded. This difficulty was overcome by eliminating the fixing step of the developing process. The unex- posed silver thus remains, and the emulsion shrinkage is reduced to only a few percent. The color shift in the reconstruction remains noticeable, but becomes tolerable. The holograms will eventually deteriorate, but have remained thus far in good condition for several months.

In private conversation, we have learned that Schwartz ${ }^{8}$ and his associates have produced holograms of the type discussed here (using one color only) and have reconstructed the hologram in white light.

We acknowledge the assistance of $R$. LaBelle in the experimental work. In addition, we acknowledge helpful discussions with E. Leith, A. Kozma and A. Friesem.

\footnotetext{
${ }^{1}$ G. Lippman, J. Phys. 3, 97 (1894).

${ }^{2}$ Y. N. Denisyuk, Opt. and Spectr. 15, 279 (1963).

${ }^{3}$ P. J. van Heerden, Appl. Optics 2, 393 (1963).

${ }^{4}$ K. S. Pennington and L. H. Lin, Appl. Phys. Letters 7, 56 (1965).

${ }^{5}$ A. Friesem, Appl. Phys. Letters 7, 102 (1965).

${ }^{6}$ H. Fleisher, P. Pengelly, J. Reynolds, R. Schools and G. Sincerbox, Proceedings of the Symposium on Optical and ElectroOptical Information Processing (MIT Press, Cambridge, Mass., 1965).

${ }^{7}$ A. S. Hoffman, J. G. Doige and D. G. Mooney, J. Opt. Soc. Am. 55,1559 (1965).

${ }^{8} \mathrm{C}$. Schwartz, Battelle Memorial Institute, Columbus, Ohio, private communication, November, 1965.
}

\section{TRANSMISSION ATTENUATION AND CONVERSION EFFICIENCY OF PROPAGATING MAGNETOSTATIC WAVES IN YTTRIUM IRON GARNET*}

(high conversion efficiency; $2^{\circ} \mathrm{C}$; ferrimagnetic material; E)

The general delay characteristics of magnetostatic waves propagating in single-crystal ferrimagnetic rods and discs have been extensively reported in the literature..$^{1-4}$ Several additional results concerning the resonant and propagating behavior of magnetostatic modes were described in a recent paper $^{5}$ that included a confirmation of predictions made by Auld $^{6}$ and an experimental determination of the limiting group velocity for low $k\left(<100 \mathrm{~cm}^{-1}\right)$ magnetostatic waves. Also, the

\footnotetext{
*This work was supported in part by the Rome Air Development Center, Griffiss Air Force Base, New York.
}

\author{
R. A. Sparks \\ Amecom Division, Litton Systems, Inc. \\ Silver Spring, Maryland \\ (Received 28 March 1966; in final form 2 May 1966)
}

behavior of the electromagnetic and magnetostatic branches (ordinary and extraordinary waves) of the dispersion diagram was investigated in the long wavelength region for a finite rod geometry.

In this Letter experimental results are presented that indicate the room temperature attenuation of magnetostatic waves is comparable with the spinwave loss characteristic of magnetoelastic wave propagation. Insertion loss measurements have been made as a function of delay time from a fraction of a microsecond up to several microseconds. The data are compared with the frequency-dependent spin-wave lifetime, $\tau_{s}$, deduced by Strauss ${ }^{7,8}$ 